\title{
Research on Influence of Community Opening on Road Capacity
}

\author{
Zi-Yu Li ${ }^{1}$, Jia-Yu Zhong ${ }^{1}$, Yuan-Biao Zhang ${ }^{1,2}$, Yi-Yan Wang ${ }^{1} \&$ Li-Qun Yang ${ }^{3}$ \\ ${ }^{1}$ Innovation Practice Base of Mathematical Modeling, Electrical and Information College of Jinan University, \\ Zhuhai, China \\ ${ }^{2}$ Key Laboratory of Product Packaging and Logistics of Guangdong Higher Education Institutes, Jinan \\ University, Zhuhai, China \\ ${ }^{3}$ HotDoor Technology Co., Ltd, Zhuhai, China \\ Correspondence: Yuan-Biao Zhang, Innovation Practice Base of Mathematical Modeling, Electrical and \\ Information College of Jinan University, Zhuhai, China. E-mail: zybt@jnu.edu.cn
}

Received: March 6, 2017 Accepted: April 2, 2017 Online Published: May 29, 2017

doi:10.5539/jms.v7n2p116 URL: http://doi.org/10.5539/jms.v7n2p116

\begin{abstract}
Under the background of promoting the block system, the paper mainly focuses on the impact on the surrounding road capacity caused by the openness of the communities. Firstly, the basic capacity model of the road is established by using Newton's second law. Then, based on the openness of the district, we analyze the probabilities of the drivers choosing the intersection and exit of the district and the delay rate of the crossing. So we establish the modified road capacity model. Finally, according to the actual situation, the road structure in the surrounding areas and intersection type is divided, and the influence coefficient of the road is introduced, which are used to establish the vehicle traffic mathematical model to quantitatively analyze whether the district opening can promote the road capacity, thus providing a certain theoretical basis for the open policy.
\end{abstract}

Keywords: community opening, road capacity, Vehicle Passing Model

\section{Introduction}

\subsection{Background Description}

Since the founding of the People's Republic of China, great progress has been made in urban planning, construction and development. However, in the process of rapid urbanization, foresight, matching and rationality of urban planning is not enough, resulting in a common problem that the city has more and more serious traffic congestion. On February 21, 2016, the State Council issued "a number of proposals on the further strengthening of urban planning and construction management", in which the sixteenth emphases optimizing the street network structure, so the new residential communities no longer build closed residential area in principle, residential quarters and the unit compound that has been completed should gradually open in order to achieve the goal of making internal road public to solve the problem of traffic.

In addition to security issues that may be caused by opening areas, the key point of the discussion is whether the open district can optimize the road network structure and improve road capacity and traffic conditions. After the opening of the district, whether the effect of the improvement of the road traffic structure is good or not should be analyzed by establishing the road capacity model of the surrounding area according to the actual situation.

\subsection{Relevant Scholarship}

Traffic capacity is one of the criteria to quantify traffic conditions. It is suitable for traffic planning, traffic management and evaluation, and the design and improvement of traffic facilities (Wang, 2013; Madanat \& Teng, 2010). Road capacity is the maximum number of traffic entities that can pass per unit time in a lane on a road $(\mathrm{pcu} / \mathrm{h} / \mathrm{ln})($ Hoban, 1987). The vehicle refers to the cars, when there are other vehicles mixed with, equivalent capacity of the standard vehicle (car) is used to be a unit (Zhang, Fang, $\mathrm{Li}, \& \mathrm{Lu}, 2015$ ). Wu improved the calculation method of traffic capacity; at the unsignalized intersection ( $\mathrm{Wu}, 2001)$. Maji used the user equilibrium model to optimize the road network flow and evaluate the intersection under the premise of the intersection (Maji \& Jha, 2010). Lan studied the impact on the intersection capacity during the rush hours in the case of signal control (Lan \& Abia, 2011). Xiao-Guang Yang etc. summarized the commonly used calculation method of intersection capacity: saturated flow rate method, stop line method, stop line method and collision point method (Yang, Zhao, Ma, \& Bai, 2014). Wang et al. analyzed the length, number of lanes, type and number 
of left-turn lanes, and the degree of road saturation of the intersections (Wang, Liu, Yu, Deng, Chen, \& Wu, 2014). An Yisheng et al. aimed at the problem that the traffic control strategy is difficult to meet the traffic flow on the input road section. Then a traffic flow optimal control model based on the time - delay Fitting Petri net is proposed (An, Lei, Yuan, \& Zhao, 2016).

\subsection{Research Design}

In this paper, according to the influence of the opening of the district on the surrounding roads, we establish the basic road capacity formula. Then the probabilities of the driver's choice of access to the intersection and the delay time of the intersection at the entrance and exit are analyzed, on which we revise the basic traffic capacity of the surrounding roads.Finally according to the unknown actual situation, the road influence coefficient is introduced and the road structure of the surrounding area is divided, so we establish the mathematical model of vehicle traffic. The structure of this paper is as follows; the second part focuses on the idea and principle of the model and the construction of the basic model; the third part, through the concrete research and analysis, establishes the vehicle traffic model based on the cell opening; the fourth part is the conclusion.

\section{Establishment of BasicModel of Road Capacity}

\subsection{Description of the Principle}

First of all, from the most ideal situation, using Newton's Second Law, we established the basic capacity of the road model before community is open. The changes of basic road capacity are a direct response to the district open to the surrounding roads. The main influencing factors of urban traffic capacity include continuous flow traffic facilities, intersection of approach roads, road conditions, environmental conditions, control conditions, etc. (Jiang, Ma, \& Jing, 2015; Chen, Gao, Xie, \& Liu, 2015; Wang, Fu, Lu, \& Han, 2016; Zhou, Pu, Zeng, \& Qiu, 2013). The impact of opening a residence community on such factors as control conditions, environmental conditions is quite little, so they are ignored in this paper.

Based on the change of the open area, the density of the road network and the area ratio of the road are increased to improve the road capacity. Therefore, the influence coefficient of the road is introduced to modify the basic road capacity. When the community is opened, the intersection increases and the delay of the vehicle at the intersection increases, so the road capacity is reduced (when vehicles cross or turn at the intersection). Whether the driver enters the residential area through intersection is a probabilistic problem. Therefore, we revised the road capacity again by studying the probability selection at the intersection in and out of the community and the length of the delay time at the intersection.

The effect of the open area is also related to the surrounding road grade and intersection type. The surrounding road structure of different areas differs from each other, including the diversity in the road grade and the types of intersection. The types of intersection to and out of the residential area can be divided into crossroads, roundabout intersections and intersections with signal lights. Therefore, the reduction coefficientof road grade and intersection delay coefficient are introduced to revise road capacityand then the capacity model based on the open community is obtained to study the impact of open residential community on the traffic around it.

We assume that the traffic capacity of the external road is $C_{\text {road }}$, and the internal road of the cell is $C_{\text {block }}$. Since the derivation of the internal and external traffic capacity in the district have some similar and different aspects, we first deduce the basic road capacity calculation, and then according to different circumstances to amend.

\subsection{Establishment of Road Basic Capacity Model}

Road capacity means the ability of a road facility to divert traffic. That is, the ability of the road facility to pass the traffic flow spot under a certain period of time (usually 15 minutes or lhour) and the normal road, traffic, control and operation quality requirements. The capacity can be divided into basic traffic capacity, potential capacity and design capacity according to the nature of traffic capacity and the use requirements (Jia, 2009). Based on this assumption, the road traffic capacity under the ideal conditions is introduced, that is, the basic road capacity.

The basic traffic capacity refers to the maximum number of vehicles crossing the road under the ideal conditions of road and traffic and the same technical performance of a standard car, with a minimum distance between the head of the ideal traffic flow in the unit time. It is also called theoretical capacity (Hu, 2012; Ma, Lu, \& Wang, 2001).

\subsubsection{Application of Newton's Second Law}

Because it is the assumed capacity under the ideal conditions. In fact, it is impossible to achieve, which can be shown with the symbol, the basic unit is $\mathrm{pcu} / \mathrm{h} / \mathrm{In}$. The formula is as follows: 


$$
C_{\text {road }}=\frac{3600}{t}=\frac{1000 v}{\lambda}
$$

In equation, in order to unify the unit, $\delta$ is equal to the quotient of unit time 3600 s divided by time $\mathrm{t}$ spent by a standard vehicle; divided by 3.6 transformation unit is $\mathrm{m} / \mathrm{s}$; for the interval distance, is:

$$
\lambda=\lambda_{1}+\lambda_{2}+\lambda_{3}+\lambda_{4}
$$

Where $\lambda_{1}, \lambda_{2}, \lambda_{3}$ separately refers to the driving distance of vehicles, braking distance and safe distance; $\lambda_{4}$ refers to the length of a standard car.

Take the safe distance $\lambda_{3}=2 \mathrm{~m}$, the standard car length $\lambda_{4}=3 \mathrm{~m}$.

Where $\lambda_{1}=v \gamma / 3.6$

Take the response time of the driver $\gamma=0.75 \mathrm{~s}$. According to Newton's Second Law, we come to the following conclusion:

$$
\begin{gathered}
2 a \lambda_{2}=\left(\frac{v}{3.6}\right)^{2} \\
\mu N=m a \\
N=m g
\end{gathered}
$$

Where $a$ refers to the accelerated speed of a car's deceleration; $\lambda_{2}$ refers to a car's braking distance in the response time; $\mu$ is to the longitudinal sliding Friction Coefficient between wheels and road; $N$ refers to supportive power; $g$ is acceleration of gravity. Moreover, the following result is:

$$
\lambda_{2}=\frac{v^{2}}{25.92 g \mu}
$$

\subsubsection{Fitting Coefficient Analysis of Friction Coefficient and Vehicle Speed}

The longitudinal sliding friction coefficient changes due to the change of running speed (Maji \& Jha, 2010), the change is shown in Table 1:

Table 1. Relationship between longitudinal sliding friction coefficient and vehicle speed

\begin{tabular}{llllllll}
\hline $\mathrm{V}(\mathrm{km} / \mathrm{h})$ & 30 & 35 & 40 & 50 & 60 & 70 & 90 \\
\hline$\mu$ & 0.45 & 0.40 & 0.35 & 0.35 & 0.30 & 0.30 & 0.25 \\
\hline
\end{tabular}

Based on the data of longitudinal sliding friction coefficient and vehicle speed in Table 1, the scatter plot is drawn by EXCEL software and the trend line is added. The fitting function is analyzed. Finally, the fitting effect of power function is the best as the Figure 1 shows:

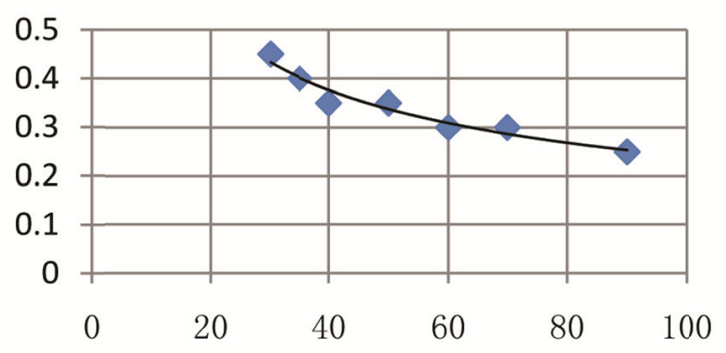

Figure 1. Relationship fitting

The resulting function is:

$$
\mu=2.2719 v^{-0.487}
$$

In the above equation, the relationship between the basic traffic capacity $C_{\text {road }}$ and the speed of the road $v$ is shown as follows: 
The relationship between the basic traffic capacity of the road and the speed change is shown in Figure 2:

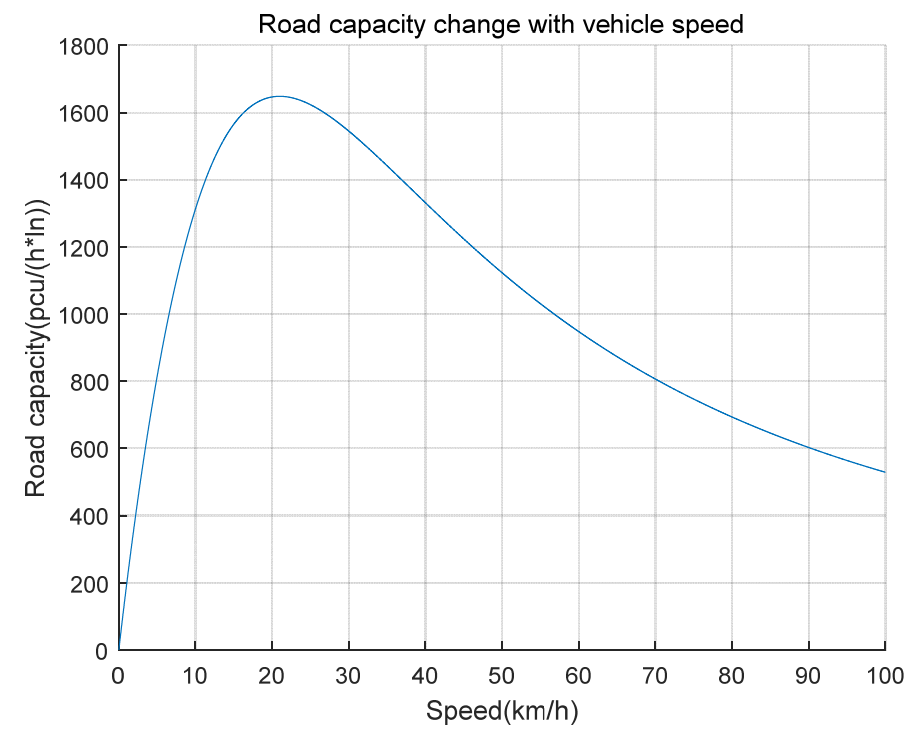

Figure 2. Road capacity change with vehicle speed

As can be seen from Figure 2, the traffic capacity of the lane is not monotonically a function of the vehicle speed. When the vehicle speed increases from $0 \mathrm{~km} / \mathrm{h}$, the road capacity increases with the increase of vehicle speed, and the road capacity reaches the highest $1648.2 \mathrm{pcu} / \mathrm{h} / \mathrm{In}$ when the vehicle speed is $21 \mathrm{~km} / \mathrm{h}$. Since then, the road capacity decreases with the increase of vehicle speed, showing a downward trend. We can summary that the greater the speed, the road capacity is not necessarily greater.

\section{Establishment of Vehicle Passing Model}

As the opening of the residential community, the choices to different road for driver increase, so here arises the question: how likely will driver choose the road inside the community instead of the main road. Besides, due to the opening of residential community, the intersections increase. Weknow that the intersections are one of the main reason that causes traffic jam, so how much of average delay time of each car crossing the intersections should be discussed in the following. Finally, basing on the above conclusion and consider the other situation like types of intersection, road structures and so on, we revise the calculation formula of road capacity. We know that and whether the vehicles of the main road around the district go in or out of the intersection of the district is a probability of choice, which will reduce the capacity of the road surrounding the community; While the road capacity of the internal structure of the district changes, that is, road network density and road area rate increases will improve the capacity of roads around the residential community, and the specific level of capacity improvement is related to the community structure and road grade.

\subsection{The Probability of Choosing a Residential Area Road at the Intersection}

In the road traffic network around the residential area, the traffic flow at the intersections of the main roads on the periphery of the community is essentially a collection of individual choice of road decisions. The road decision is a comprehensive reflection and influence of the driver in a specific traffic environment. There are many main factors for the driver to choose the intersection of an open area, such as passage time, passage cost, and timedelay, road condition, crowding degree, community environment and weather conditions. There are three criteria for the driver to choose a path's decision-making behavior (Liu, 2009), which is the shortest time utility, the shortest distance utility, the lowest economic efficiency.

For different types of drivers, there are some differences of the behavior criteria, so the drivers are divided into two types: high-risk drivers who may choose other roads in congested situations and the conservative drivers of the high probability of choosing the trunk in any case. The standard of classification is related to the driver's familiarity with road conditions, driving experience, the driver's character and other factors.

According to the actual situation analysis, when driving at a low speed (such as $0 \sim 15 \mathrm{~km} / \mathrm{h}$ ), most adventurous drivers will choose the internal branch of the open district to avoid traffic congestion, but most conservative drivers are willing to travel in the main road, therefore, at a low driving speed, the probability for all drivers to choose the main road is low, but because of the existence of the conservative drivers, the probability will not be 
lower than a certain level.

When the speed is higher than the driver's minimum satisfactory speed, very few drivers will choose the road in the residential area. As the driving speed gradually increases, that is, when the road level is improved, the probability of the driver selecting the main road will be increased at a higher speed because the driving speed is higher than the minimum satisfactory speed of the driver.

When the driving speed is at a high level, that is, in good road conditions, the main road will be the best choice in a certain speed range (such as $60-80 \mathrm{~km} / \mathrm{h}$ ), so the probability for the drivers to choose the main road will remain at a high level and tend to be stable.

To sum up, the driving speed $\mathrm{v}$ is chosen as the driver selection criteria on the intersection of the main road around the district and out of the district, and the large Cauchy distribution fuzzy function is chosen to be the membership function of probability selection. The large Cauchy distribution function is as follows:

$$
f(x)= \begin{cases}{\left[1+\alpha(x-\beta)^{-2}\right.} & \mathrm{c} \leq x \leq e \\ a \operatorname{In} x+b & \mathrm{e} \leq x \leq f\end{cases}
$$

Among them $\alpha, \beta, a, b$ are undetermined coefficients. According to the actual situation, the average walking speed of a person is $4.5-6 \mathrm{~km} / \mathrm{h}$, when the road congestion is very serious, the speed may be $5 \mathrm{~km} / \mathrm{h}$, and the probability of choosing the main road around open area is 0.5 , When the road speed is $30 \mathrm{~km} / \mathrm{h}$, the probability is 0.9 ; when the condition of surrounding main road is better, and the road is very smooth, if the speed is $80 \mathrm{~km} / \mathrm{h}$, the probability is 0.99 . The available probability function is as follows:

$$
P(v)=\left\{\begin{array}{lc}
{\left[1+156.25(v+7.5)^{-2}\right]^{-1}} & 5 \leq v \leq 30 \\
0.092 \operatorname{In} v+0.588 & 30 \leq v \leq 80
\end{array}\right.
$$

After the residential community is open, the probability of choosing the main road around is shown in Figure 3:

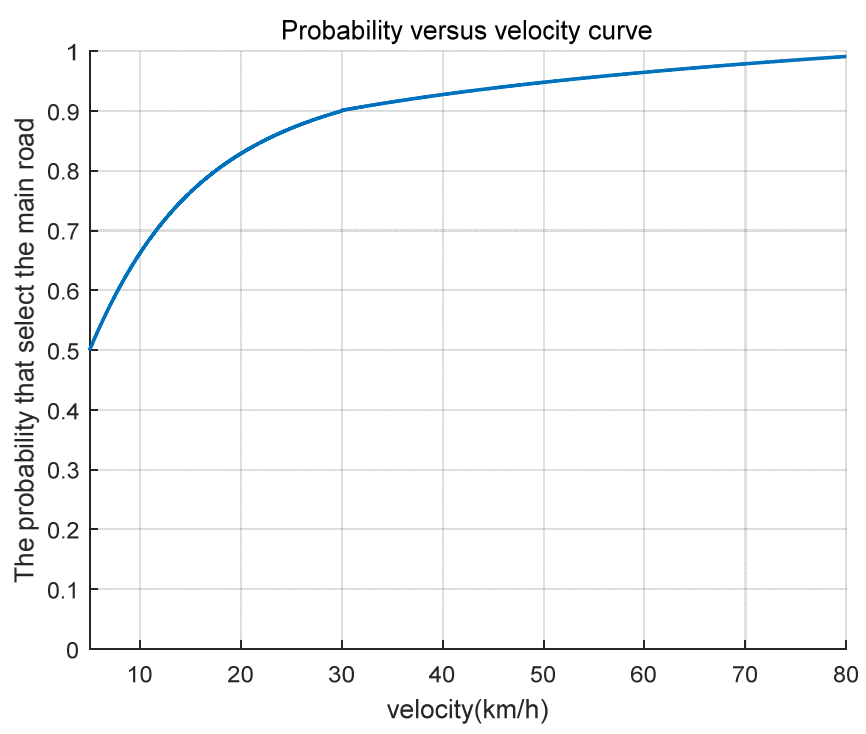

Figure 3. Probability versus velocity curve

Obviously, the probability of choosing a residential area road at the intersection is $1-P(v)$.

\subsection{The Average Delay Time at the Intersection in and out of the Community}

At the intersection in and out of the community, road capacity will be reduced because traffic vehicles need to pay attention to the left and right side or the vehicles in front need to slow down when turning, thus the intersection of the community will reduce the road capacity.

If there is no car in a certain distance between the front and the rear of the vehicle under the condition of smooth traffic flow and unimpeded traffic, the decline of the road capacity due to slowing down or safe driving at the 
intersection can be ignored.

As the number of vehicles increases, the vehicle speed decreases, and when the front vehicle decelerates, the rear follow-up vehicle also decelerates, and the more cars follow, the higher the accumulated delay is. Therefore, with the increase in traffic flow, the delay at intersection will increase at a higher rate.

It may be assumed that the delay in access to the cell is a function of the speed of the vehicle, and the average delay in the access to the information (Wang, 1998) is shown in Table 2:

Table 2. Average delay of the intersection

\begin{tabular}{ll}
\hline Vehicle average delay (s) & Traffic condition description \\
\hline$<=5.0$ & Traffic smooth, basically unimpeded \\
$5.1 \sim 15.0$ & Traffic is basically smooth, slightly resistance \\
$15.1 \sim 30.0$ & Traffic is running normally, there is a certain delay \\
$30.1 \sim 50.0$ & Traffic can still run normally, but the delay has been larger \\
$50.1 \sim 80.0$ & Traffic has been in a crowded state, a great delay \\
$>80.0$ & Traffic has been supersaturated, often in a blocked state \\
\hline
\end{tabular}

Take the speed of $80 \mathrm{~km} / \mathrm{h}$ for the traffic flow is unobstructed, $50 \mathrm{~km} / \mathrm{h}$ for the traffic flow is basically smooth, $40 \mathrm{~km} / \mathrm{h}$ for normal traffic, $20 \mathrm{~km} / \mathrm{h}$ is still normal operation, $10 \mathrm{~km} / \mathrm{h}$ has been in a crowded state, $5 \mathrm{~km} / \mathrm{h}$ when the traffic has been supersaturated. Using the scatter plot to match the average delay time of the vehicle on the speed of the function:

$D(v)=105.91 e^{-0.046 v}$

Based on the above discussing results, we conclude that the road capacity inside the community is:

$$
C_{\text {block }}=\frac{3600}{\lambda /(v / 3.6)+D(v)} \cdot(1-P(v))
$$

\subsection{Improvement of calculation formula of traffic capacity of surrounding roads}

To consider the simplest case, as shown in Figure 4:

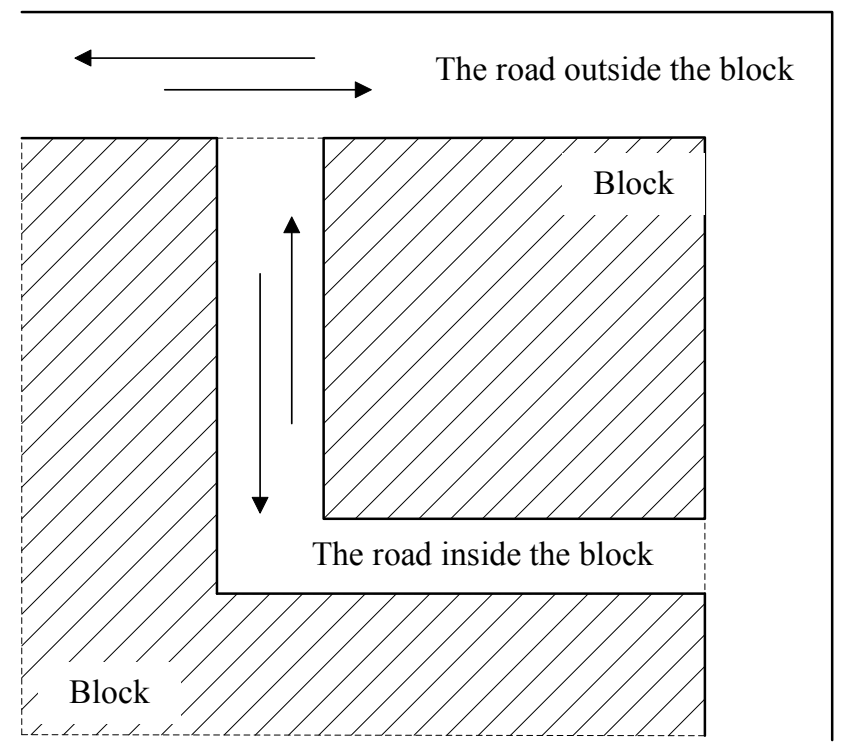

Figure 4. Simple cell schematic

Assuming that the length of the road within the residential area is equal to the length of the original road outside the community as well as width and the road grade. After the block open, the road capacity will be simply sum up as shown in Figure 4. 


$$
C_{\text {all }}=C_{\text {road }}+C_{\text {block }}
$$

However, in the actual situation, the traffic conditions, regulatory conditions, environmental conditions and road conditions are different in the traffic. In the amendment of the road capacity formula, the effect of district openness is mainly considered, that is, the regulatory conditions and environmental factors are ignored. Due to the diversity of the internal structure of the district and on this basis, the traffic capacity of the surrounding roads is amended. And the influence coefficient of the road $\vartheta$ is introduced and the influence degree of the district opening on the surrounding road traffic is characterized. The formula of the modified road capacity is as follows:

$$
C_{\text {all }}^{*}=C_{\text {road }}+C_{\text {block }} \cdot \vartheta
$$

As the influence coefficient $\vartheta$ vary with different road structure in various block, we assume that $\vartheta=1$.The relationship between the modified road traffic capacity varies with the speed is shown in Figure 5.

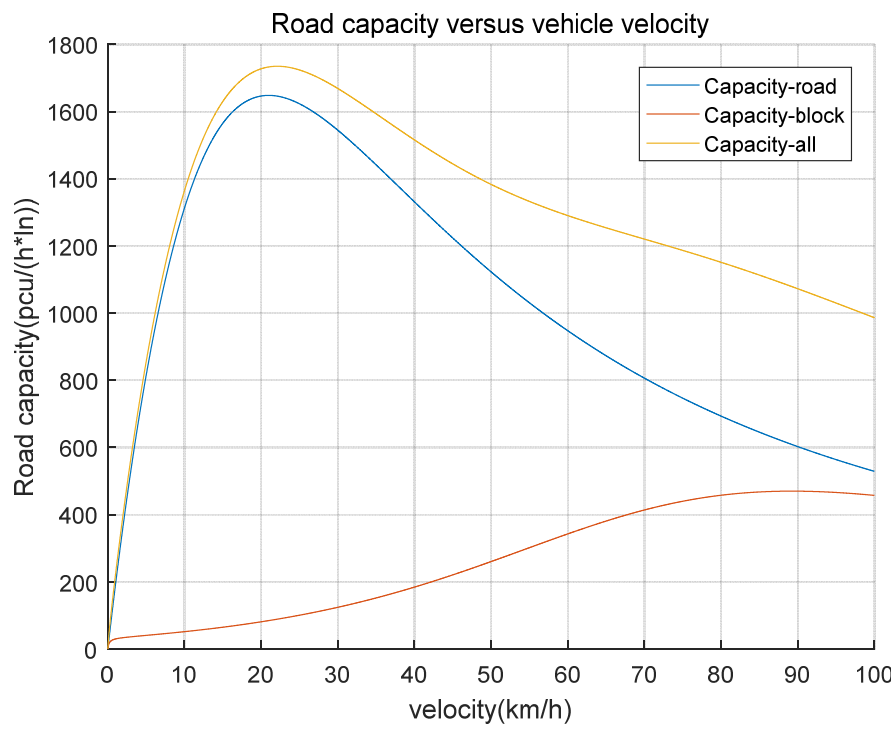

Figure 5. Modified road capacity versus vehicle velocity

\subsection{Influence of the Surrounding Road Structure}

In the case of the determined plot type and certain traffic flow level, the influence of the surrounding road structure on the road capacity after the opening of the community mainly lies in two aspects:

First: the road grade. The speed of vehicles in different grades of road is not the same. The higher the level of the road, the faster the average speed is;

Second: the type of intersection around the district. Because of the existence of different intersections, the average delay time for drivers through the intersection is also different.

\subsubsection{Road grade reduction factor $r$}

The lane width has a great influence on the driving speed. The wider the lane is, the greater the road capacity is, that is, the higher the road grade is, the greater the road capacity is. The standard lane width of is $3.5 \mathrm{~m}$ in China and if the width of the lane is wider than $3.5 \mathrm{~m}$, it will help to increase the speed of the vehicle. On the other hand, if the lane width is less than $3.5 \mathrm{~m}$, the driving speed will be limited. The basic road capacity around the area is revised according to the amendment of road grade information (Ministry of Construction of the People's Republic of China, 1995), and the correction results are shown in Table 3 below:

Table 3. Road grade reduction factor

\begin{tabular}{llllllll}
\hline Road type & Freeway & Trunk road & \multicolumn{2}{l}{ Secondary road } & \multicolumn{2}{l}{ Branch } \\
\hline Classification & 1 & 2 & 3 & 4 & 5 & 6 & 7 \\
Lane width & $>=3.5 \mathrm{~m}$ & $3.5 \mathrm{~m}$ & $3.0 \sim 3.25 \mathrm{~m}$ & & $2.75 \sim 3.0 \mathrm{~m}$ \\
Reduction factor & 1.00 & 1.00 & 0.94 & 0.85 & 0.85 & 0.85 & 0.77 \\
\hline
\end{tabular}




\subsubsection{Intersection Delay Coefficient $\sigma$}

When the residential area is opened, there will be such traffic flow as conflict, intersection and diversion caused by steering at the road junctions on and off the main road around the residential area, making the traffic characteristics of intersections complex. The traffic capacity of intersections varies according to the vehicle Traffic and cell structure will have an obvious difference.

According to the different ways of control to intersections, road junctions can be divided into circular intersections, uncontrolled intersections and signalized intersections. The delay degree at the three types of intersection is discussed as follows:

1) In the case of the same district structure, the same road structure and the same traffic flow, when the traffic flow at the intersection is very small, vehicle can pass directly at no control intersection, so it's the smallest delay, and the delay is maximum at signalized intersection due to the existence of traffic lights, and the delay at the circular intersections is between the two.

2) With the increase of traffic flow, traffic congestion often happen at uncontrolled intersections because of the lack of corresponding traffic control measures, so the delay degree at uncontrolled intersections increases fastest. The existence of traffic control can effectively relieves traffic congestion at signalized intersection, and its delay grows the most slowly; the roundabout can alleviate part of the traffic congestion due to the existence of a greater detour distance, so its delay is between the two.

According to the above analysis, the delay of the three kinds of intersection on the traffic flow curve (Wang, 1998) is shown in Figure 6:

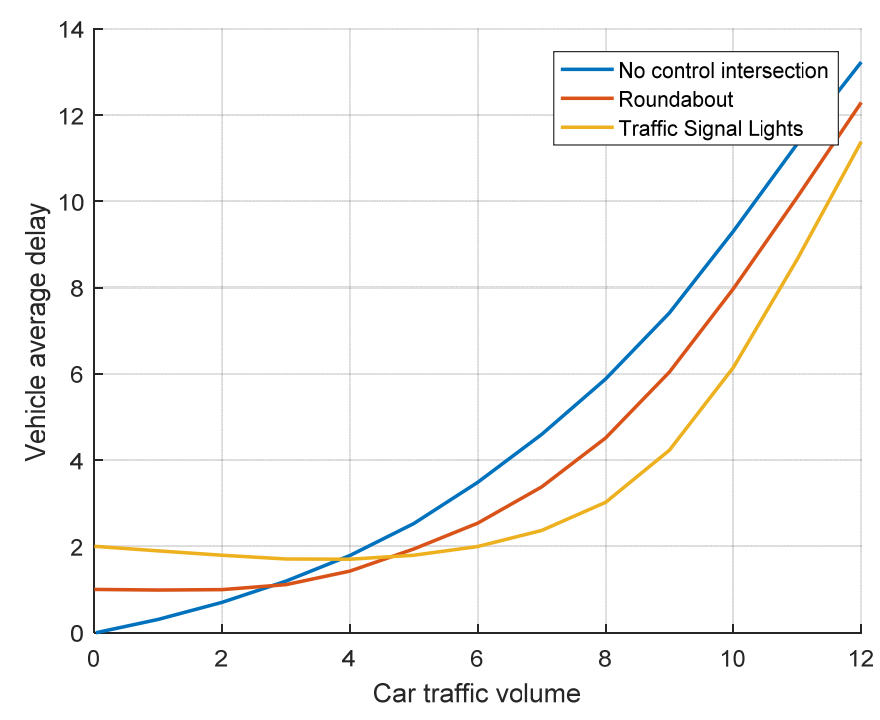

Figure 6. Different types of intersections delay-traffic flow

On the basis of this, we can link the traffic flow to the speed. The traffic capacity of the surrounding roads after the opening of the community is revised once again according to the unknown actual situation, and the final road capacity is obtained as follows:

$$
C_{\text {final }}=C_{\text {road }} \cdot r_{1}+C_{\text {block }} \cdot \vartheta \cdot r_{2} \cdot \sigma
$$

\section{Conclusion}

From the closed area to the street system of policy transformation process, it was closely related to sustainable development of the city and ecological development of the district. The policy pointed out that the purpose of optimizing the street network structure was to solve the problem of traffic network layout, promote land conservation, construct reasonable road network system, which led to a lot of discussion of public space. This article focuses on the impact of open residential area on the surrounding roads, that is, whether the open community can improve the traffic capacity of the surrounding roads to achieve the purpose of improving the traffic conditions. 
Under the background of this urbanization, this paper first uses the Newton's Second Law to derive the basic road capacity formula. Through analyzing the probabilistic problem of the driver's choice of access to the intersection and the delay time of the intersection, we revise the basic traffic capacity of the surrounding roads. Then according to the unknown actual situation, the road influence coefficient is introduced and the road structure of the surrounding area is divided, Bases on it, we establish the vehicle passing model.

After opening, it will not simply increase the capacity of its surrounding roads, which is related to the driving speed of the driver, the structure of the open area inside the community, the road grade of the surrounding area and the type of intersection and its delay rate. The vehicle traffic model quantitatively evaluates the traffic capacity of the open roads, and provides a theoretical basis for the open policy of the community so that the urban construction can develop better and faster.

\section{References}

An, Y. S., Lei, T., Yuan, S. X., \& Zhao, X. M. (2016). Traffic signal control model and optimization based on timed colored Petri nets. Application Research of Computers, (1), 194-198.

Chen, L., Gao, J., Xie, G., \& Liu, H. (2015). Routing to enhance traffic capacity for scale-free networks with tunable clustering. IEEE Advanced Information Technology, Electronic and Automation Control Conference (pp. 110-113). IEEE. https://doi.org/10.1109/iaeac.2015.7428529

Hoban, C. J. (1987). Procedures for evaluating traffic capacity, and improvements to road geometry. Geometric Segments.

Hu, M. W. (2012). Traffic Engineering. Bei Jing: China Quality Inspection Press.

Jia, X. M. (2009). Research on Influence Factors of Urban Road Capacity. (Doctoral dissertation, Chang'an University).

Jiang, Z. Y., Ma, J. F., \& Jing, X. (2015). Enhancing traffic capacity of scale-free networks by employing hybrid routing strategy. Physica A Statistical Mechanics \& Its Applications, 422, 181-186. http://dx.doi.org/10.1016/j.physa.2014.12.016.

Lan, C. J., \& Abia, S. D. (2011). Determining peak hour factors for capacity analysis. Journal of Transportation Engineering, 137(8), 520-526. http://dx.doi.org/10.1061/(ASCE)TE.1943-5436.0000241.

Liu, Y. H. (2009). The Research of the Drivers Route Choice in the Traffic Information. (Doctoral dissertation, Southwest Jiaotong University).

Ma, J. X., Lu, Z. Y., \& Wang, D. M. (2001). The Analysis and Improvement on Capacity of Urban Road. Journal of Nanjing Forestry University (Natural Science Edition), 25(2), 71-74.

Madanat, S., \& Teng, H. (2010). Transportation research board, national research council, trb. Ite Technical Report, 24(Feb).

Maji, A., \& Jha, M. K. (2010). Considering intersection performance in road network flow optimization using user equilibrium approach. Wseas International Conference on Computer Engineering and Applications (pp. 207-211). World Scientific and Engineering Academy and Society (WSEAS).

Ministry of Construction of the People's Republic of China. (1995). Code for Urban Road Traffic Planning and Design. Bei Jing: China Planning Press.

Wang, S. (2013). Research Status and Development Trend of Urban Road Capacity. Theoretical Study on Urban Construction: Electronic Edition, (18).

Wang, W. (1998). Analysis method for capacity of road junctions based on the delay-curve. China Journal of Highway \& Transport.

Wang, X., Fu, H., Lu, J., \& Han, S. (2016). Study on road section environmental traffic capacity model and algorithm under double constraints. Transportation Research Part D Transport \& Environment, 48, 14-19. http://dx.doi.org/10.1016/j.trd.2016.07.005.

Wang, X., Liu, H., Yu, R., Deng, B., Chen, X., \& Wu, B. (2014). Exploring operating speeds on urban arterials using floating car data: case study in shanghai. Journal of Transportation Engineering, 140(9), 04014044. http://dx.doi.org/10.1061/(ASCE)TE.1943-5436.0000685

$\mathrm{Wu}, \mathrm{N}$. (2001). A universal procedure for capacity determination at unsignalized (priority-controlled) intersections. Transportation Research Part B Methodological, 35(6), 593-623. http://dx.doi.org/10.1016/S0191-2615(00)00012-6. 
Yang, X. G., Zhao, J., Ma, W. J., \& Bai, Y. (2014). Review on Calculation Method for Signalized Intersection Capacity. China Journal of Highway and Transport, 27(5), 148-157.

Zhang, X. R., Fang, Z. X., Li, Q. Q., \& Lu, S. W. (2015). A Spatio-temporal Analysis on the Heterogeneous Distribution of UrbanRoad Network Capacity Based on Floating Car Data. Journal of Earth Information Science, 17(3), 336-343.

Zhou, F. Y., Pu, L. L., Zeng, F. Y., \& Qiu, X. P. (2013). Research Status and Development Trend of Urban Road Capacity. Highways \& Automotive Applications, (1), 32-36.

\section{Copyrights}

Copyright for this article is retained by the author(s), with first publication rights granted to the journal.

This is an open-access article distributed under the terms and conditions of the Creative Commons Attribution license (http://creativecommons.org/licenses/by/4.0/). 